\title{
Homo quadratus \\ Variations sur la beauté et la santé dans la médecine antique
}

Par Jackie Pigeaud

Au début du $2^{\mathrm{e}}$ livre de son De medicina, Celse écrit: Corpus autem habilissimum quadratum est, neque gracile neque obesum. «Or le corps le mieux adapté est carré, et il n'est ni maigre ni obèse.» ${ }^{1} \mathrm{~J}$ 'aimerais dédier au penseur qui a su si profondément unir la réflexion médicale, la littérature et l'esthétique, quelques rêveries philologiques dont la phrase de Celse sera le prétexte.

Donc Celse donne quelques indications sur ce que doit être l'apparence du corps pour être sain. Il continue en écrivant: Nam longa statura, ut in iuuenta decora est, sic matura senectute conficitur, gracile corpus infirmum, obesum hebes est. «Car une haute taille, si elle a de la beauté dans la jeunesse, s'altère dans la plénitude de l'âge, un corps maigre est faible, un corps obèse est sans vivacité.» Il y a belle lurette que les érudits ont reconnu, dans cette dernière assertion, un Aphorisme d'Hippocrate (II, 54 - IV L 486) «Une haute taille, dans la jeunesse, est noble et non sans grâce; mais dans la vieillesse, elle est plus embarrassante et moins avantageuse qu'une taille moindre. ${ }^{2}$

Il n'y aurait, à première vue, pas grand'chose à dire de tout cela. Mais ce n'est pas si simple. Que signifie exactement quadratus? Il semblerait que quadratus renvoie à la statura, si l'on en croit le parallélisme de l'explication qui suit: une haute taille a tel défaut, le corps maigre et le corps obèse tels autres défauts. Ce qualificatif de quadratus évoque le portrait de Vespasien par Suétone ${ }^{3}$, qui parle de la statura quadrata du Prince, et il n'est nullement exclu, chez un auteur qui s'intéresse, comme lui, à la physiologie, que ce soit un souvenir de Celse. Naturellement aussi l'on songe à Vitruve et à l'inscription qu'il fait du corps humain idéal dans le carré et dans le cercle ${ }^{4}$. Naturellement encore l'on pense au pasşage où Pline décrit les statues de Polyclète. «Une caractéristique de sa manière, c'est d'avoir imaginé de faire reposer ses statues sur une seule jambe; pourtant Varron dit qu'elles sont carrées et presque toutes sur le même modèle.» Et naturellement, Polyclète étant l'auteur du traité et de la statue que l'on appelle la Règle, le Canon, sans doute le Doryphore, l'on voit s'imposer une sorte de canon physique et 
physiologique. L'on circulerait ainsi de façon «naturelle» entre l'art et la médecine, les choses allant de soi, puisqu'il nous semble vraisemblable que la médecine prête au sculpteur une connaissance du corps humain, tandis que le sculpteur offre au médecin une forme idéale de ce corps.

Cette forme carrée du corps a-t-elle quelque chose à voir avec une idée esthétique, et même avec le fameux canon de Polyclète? C'est ce qu'Overbeck lui-même semblerait entendre, puisqu'il cite Celse à la suite du passage de Pline ${ }^{5}$. Peut-être Celse se souvient-il seulement d'un chapitre de Prorrhétique II, 7 (IX L 25). «On louera une poitrine carrée $(\tau \varepsilon \tau \varrho \alpha ́ \gamma \omega \nu o v)$ et velue; le cartilage en sera petit et bien garni en chair» (trad. Littré) ${ }^{6}$. Y a-t-il déjà une idée «esthétique» sous-jacente dans le Prorrhétique II? Se pourrait-il même qu'il y en eût une? ${ }^{7}$ Nous allons nous demander si ces accords entre un sens esthétique et un sens médical vont tellement de soi. Le problème que nous voulons poser dépasse le sens de quadratus. Qu'en est-il des relations entre l'art et la médecine dans l'Antiquité, du rapport entre la Beauté et la Santé? Bien évidemment nous ne saurions qu'esquisser ici un tel sujet.

En vérité, dans ces problèmes où l'on rencontre tant d'implicite, il n'est pas facile d'y voir clair. Quelles sont les rapports entre le corps vu par le statuaire et le corps imaginé par le médecin; disons, pour parler approximativement, entre Polyclète et Hippocrate? De quelle façon l'art et la médecine ont-ils pu s'influencer; et comment l'idée de l'art a-t-elle pénétré la médecine? Questions redoutables que nous essayerons seulement d'approcher aujourd'hui ${ }^{8}$. Nous commencerons par Galien. Après tout l'on doit noter que c'est lui qui nous donne les plus complets renseignements sur le Canon; et l'on s'aperçoit que Polyclète tient un rôle central dans la réflexion galénique ${ }^{9,10}$.

Les deux témoignages principaux que nous donne Galien ne sont pas dans les mêmes contextes et changent de signification, relativement aux problèmes qui nous occupent. Dans les Dogmes d'Hippocrate et de Platon ${ }^{11}$, Galien reproche à Chrysippe, à propos de l'âme, d'avoir confondu la beauté et la santé. Il n'a pas raisonné pour l'âme, comme il l'a fait pour le corps, en distinguant les parties et les éléments. Laissons de côté le problème de l'âme ${ }^{12}$, et considérons ce qui est dit du corps. Chrysippe, en ce qui concerne le corps, a bien raisonné en définissant la beauté comme proportion (symmetria) des parties et la santé comme proportion (symmetria) des éléments. Ainsi la santé est proportion entre chaud, froid, sec et humide qui sont les éléments; tandis que la beauté est proportion du doigt au doigt, de tous les doigts à la paume et au poignet, de ces parties à l'avant-bras, de l'avant-bras au bras, et

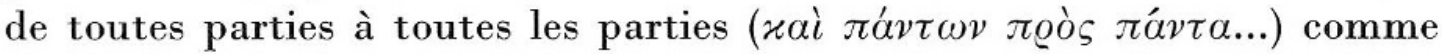


Polyclète l'a écrit dans son Canon. «Polyclète le premier, dit Galien, nous a donné un enseignement dans ce livre sur les proportions du corps et il confirma cet enseignement en acte, en fabriquant une statue en accord avec les postulats de sa théorie; et il donna à cette statue, comme au traité, le nom de Canon.» Très intéressant est ce que souligne aussitôt Galien. Tous les médecins et tous les philosophes, sans exception, placent la beauté du corps dans les proportions des parties du corps, et la santé dans les proportions des éléments. Tous, c'est-à-dire, la liste est intéressante, Asclépiade, Epicure, Anaxagore, Chrysippe et les Stoïciens, Aristote et Théophraste, Platon et Hippocrate. Il y a là une unanimité exceptionnelle puisque nous y trouvons les exclus habituels de tout consensus que sont les atomistes; cette unanimité fait fi, comme le dit Galien, de ce que chacun met sous le nom d'éléments du corps (atomes, homoeomères, humeurs, peu importe). La distinction entre beauté et santé est unanimement reconnue et leur définition aussi. Ainsi la définition du beau par Polyclète comme proportion des parties entre elles peut-elle être acceptée de quiconque ${ }^{13}$.

La finalité de l'artiste est évidemment la beauté, c'est-à-dire la proportion des parties; celle du médecin, la santé, c'est-à-dire la proportion des éléments. L'on peut réfléchir à cette disparité essentielle. Elle est fondée, pourrait-on dire, aussi bien historiquement que philosophiquement. L'artiste s'est préoccupé des parties constitutives du corps humain et de ses articulations, mais de manière tout à fait externe. Le médecin s'est peu soucié, à l'origine, d'une description exhaustive des parties, et ne fonde pas sa science sur une telle conception.

Le raisonnement du passage du De temperamentis où intervient encore Polyclète est beaucoup plus complexe. Galien prend pour point de départ de sa réflexion la définition du tempérament moyen. Le médecin s'exercera à concevoir les bons mélanges en chaque genre, et les natures moyennes, ce qui revient au même; et l'on devra juger des tempéraments par écart avec cette moyenne qui fonctionnne comme norme ${ }^{14}$. Pour parvenir à la moyenne, l'on partira par exemple du plus chaud (que les sens nous donnent sous la forme du feu ou de l'eau bouillante), vers le plus froid (glace ou neige), et, par la pensée, l'on obtiendra un symmetron, une mesure, une proportion, à égale distance de chaque extrême. On peut réaliser ce «symmetron» dans l'expérience, en mélangeant une quantité de glace à une même quantité d'eau bouillante, et en touchant le mélange. L'on gardera ensuite la mémoire de cette sensation, et l'on s'y référera comme à un canon ${ }^{15}$. L'on prendra donc comme référence, comme règle (canon) et comme norme (gnômon) un type 
moyen par rapport auquel on jugera le reste comme chaud, froid, sec ou humide. Les modélistes, les dessinateurs, les statuaires et autres fabriquants de reproductions, reproduisent le plus beau à l'intérieur de chaque espèce, en cherchant le milieu, la moyenne entre chaque espèce. «Et l'on fait ainsi l'éloge d'une statue de Polyclète appelée Canon qui a reçu ce nom du fait qu'elle a la proportion (symmetria) la plus exacte de toutes les parties les unes à l'égard des autres.»

Le lien des idées peut paraître obscur. C'est que nous avons la notion de mélange, ce que signifie le terme même de crase (tempérament); nous sommes là dans le domaine des humeurs, des éléments dont nous parlions naguère. Galien conçoit ensuite un mélange moyen, fait d'égalité entre les éléments; puis pose cette égalité, cette moyenne, comme norme et comme mesure. Cette idée régulatrice évoque le Canon de Polyclète que cite ici Galien. Chose très intéressante, ce glissement ajoute un caractère nouveau à la norme polyclitéenne, celui de moyenne. L'homme de Polyclète, le Doryphore, serait l'homme moyen. Le Doryphore représenterait le meilleur de l'espèce humaine, parce que Polyclète aurait pris la moyenne de cette espèce $^{16}$. Le Doryphore comme moyenne et comme idéal, la stature carrée typique de Polyclète, comme nous le dit Pline, pourrait s'harmoniser avec une interprétation de ce type du quadratus de Celse. Puis Galien continue en disant que le canon qu'il recherche est plus difficile que celui de Polyclète. L'homme ainsi «bien en chair» (eusarkos, terme que le traducteur de la Renaissance transpose immédiatement en carré, quadratus) qu'envisage le médecin, doit avoir en même temps la moyenne entre l'humide et le sec, et être formé de la meilleure façon ${ }^{17}$. En vérité, dit Galien, la mesure, la proportion (symmetron) dans le «bien en chair», l'embonpoint (eusarkos) prend sa naissance dans le bon mélange, le mélange bien proportionné (eucrasie $)^{18}$. L'on voit que la symmetria ne renvoie pas seulement à la proportion entre les parties, mais qu'ici s'esquisse une symmetria des éléments, une proportion entre les éléments du mélange qui forme la crase; et que Galien rêve à une sorte de Polyclète du tempérament. Le canon médical serait celui qui concilierait le rapport des parties entre elles et le rapport entre les éléments, étant bien entendu, en dernière analyse, que ce sont les éléments qui secrètent finalement la forme. En fait, Galien rêve ici de concilier deux idées de la création.

Il faut comprendre, en vérité, que la médecine propose face à une idée de la création comme re-production, une idée de la création comme production ${ }^{19}$. «Il y a deux manières de façonner $(\delta \iota \alpha \pi \lambda \alpha \tilde{\tau} \tau \varepsilon \iota \nu)$ avec art: ou bien on 
touche extérieurement l'objet, ou bien on le pénètre tout entier» ${ }^{20}$, écrit Galien dans un autre contexte. Il est évident que le statuaire doit se contenter de la première façon. Il ne saurait que re-produire. «Un Praxitèle, un Phidias... se bornent à façonner la matière extérieure, celle qu'on peut toucher; quant à la partie profonde, ils la laissent privée d'ornements, brute... incapables qu'ils sont d'y pénétrer, d'y descendre et de toucher toutes les parties de la matière.» ${ }^{21}$ La Nature au contraire, touche, finit et orne l'intérieur du corps. Reproduire, c'est assembler des parties reconnaissables, identifiables, mesurables, peut-être commensurables, dans un matériau qui n'est pas celui de l'objet reproduit, en un certain rapport qui permet d'identifier un ensemble, et d'apprécier sa justesse par un jugement qui va de la partie au tout et du tout à la partie, cette justesse pouvant s'appeler pour certains la beauté. Il va de soi que le matériau lui-même ne saurait être modifié dans sa substance. Produire, c'est pénétrer une matière de façon à l'altérer complètement, à la transformer, à la transmuter. Le matériau ne saurait rester identique. Mais la nature du sang fera la chair, l'os, les muscles, etc. Le sang n'est pas la cire, «matière simple et à figure unique sur laquelle s'exerce l'artiste» ${ }^{22}$. Et pourtant former, ce n'est pas seulement transmuter; c'est aboutir à des formes, à des parties reconnaissables, identifiables, à l'intérieur comme à l'extérieur du corps humain; ce qui repose le problème esthétique des rapports et de la justesse de ces rapports. L'Antiquité a poussé ces deux réflexions; l'esthétique de la reproduction fait trop oublier l'esthétique de la production. En quelque sorte, aucune réflexion esthétique ne saurait se dispenser de Galien; et l'esthétique de Galien n'est pas une fantaisie culturelle du médecin, mais se trouve au cœur même de sa réflexion de médecin. Elle est, ce qu'il appelle sa théologie. C'est pourquoi l'exemple de Polyclète est très précieux chez Galien, comme analogon et comme contreépreuve.

Le créateur ne peut agir comme le Dieu de Moïse. «Pour Moïse, il suffit que Dieu ait voulu orner la matière et soudain la matière est ornée; car il pense que tout est possible à Dieu, voulût-il même, avec de la cendre, faire un cheval ou un bœuf. Pour nous, nous ne jugeons pas ainsi, mais nous prétendons que certaines choses sont impossibles à la nature; que Dieu, loin d'en faire l'épreuve, se contente parmi les choses possibles de choisir les meilleures.» ${ }^{23}$

Idée capitale. D'abord jamais les Grecs n'ont cru à la création ex nihilo. Tout créateur, fût-il Dieu, crée à partir d'une matière pré-existante. Mais en plus, il existe une résistance de cette matière, qui exige un temps de fabrication de l'objet, et une manière de prendre et de jouer avec le 
matériau ${ }^{24}$, dont la nature ne saurait se dispenser par décret. En quoi la nature, en quelque façon, se retrouve dans les mêmes conditions que Phidias ou Polyclète.

La nature se préoccupe de la beauté, dans tous les aspects de sa définition, c'est-à-dire qu'elle ne se soucie pas seulement, par ordre d'importance, de l'utilité, et de la fonction, qui sont pour Galien les deux composants les plus importants du beau, mais aussi de l'ornement, du fini comme nous dirions. Ainsi pour les oreilles, le prépuce, les lèvres du sexe féminin, la chair des fesses. «La nature s'occupe par surcroît de ce soin, ne laissant sortir de ses mains aucune partie sans lui avoir donné tout son poli, son fini, son harmonie. De même, en effet, que les ouvriers habiles pour fournir, en dehors de leur travail, un échantillon de leur savoir faire, se plaisent soit sur des couvercles et des boucliers, soit sur la poignée des glaives... à ajouter quelque ornement étranger à l'utilité de l'objet et emprunté à la statuaire, ils y cisèlent des feuilles de lierre, ... de même la nature a par surcrô̂t embelli tous les membres, principalement ceux de l'homme...» ${ }^{25} \mathrm{Si}$, comme le rappelle Galien, dans un langage qui pourrait bien être stoïcien ${ }^{26}$, «la beauté véritable renvoie à la perfection de l'utilité, et si la première visée de toutes les parties est l'utilité de leur disposition», la nature ne néglige pas le détail inutile mais qui fait plaisir à l'œil ${ }^{27}$, se faisant non plus sculpteur mais ciseleur ${ }^{28}$.

Bien évidemment, Galien pense trouver chez Hippocrate une théorie de la mesure, de la proportionnalité, de la symmetria. Toute la question est de savoir s'il a raison de l'y rencontrer.

Galien, attentif à l'équitable répartition des parties du corps, loue par exemple Hippocrate d'avoir appelé la nature juste ${ }^{29}$. Dans l'esprit de Galien il s'agit d'une justice distributive qui donne à chaque organe son rôle à l'intérieur du corps. Il est vrai qu'Hippocrate utilise l'adjectif díxaıs. Erotien avait aussi éprouvé la nécessité de le gloser ${ }^{30}$. Si l'on étudie de près les emplois de l'adjectif, ce que nous ne saurions faire ici, l'on s'aperçoit qu'on peut le rendre souvent par l'idée de convenance, de naturel, d'approprié, de «normal», si l'on peut risquer ce terme sans lui donner un sens transcendant, de correct, de régulier. Par exemple, il est question de la «juste» disposition du membre (Fractures 30 = Pétrequin II, 196), de l'application correcte d'un levier (Articulations 7 = Pétrequin II, 298). Dans la pratique de l'art, il faut que le médecin retrouve, dans chaque cas, les attitudes naturelles, les positions justes des membres ${ }^{31}$. La justice peut aussi représenter ce à quoi l'on peut s'attendre, la normalité au sens le plus plat du terme. Par exemple Epidémies VI, 4, 22 (V L 312): «Tels sont les yeux, tel le corps; la couleur 
empire ou s'amende; il est «naturel» (juste), que l'extérieur suive les qualités de la nourriture.»

En vérité, il est bien difficile de donner à cette justice hippocratique le sens philosophique que Galien voudrait lui attribuer. Quand Hippocrate, à propos du pied bot, parle de «juste nature» des parties à laquelle il faut ramener ce pied en le modelant comme de la cire, il est difficile de voir autre chose, en cette juste nature, que la disposition naturelle ${ }^{32}$. Il est bien difficile de tirer cette idée de «justice» vers la mesure et les rapports, sauf peut-être en un passage d'Articulation $10=$ Pétrequin II, 310, que je donne dans la traduction de Pétrequin: «On reconnaîtra qu'un bras s'est luxé à l'aide des signes suivants; et d'abord, comme les hommes ont le corps symétrique (c'est ainsi que Pétrequin traduit díxaıs), pour les membres tant supérieurs qu'inférieurs, il faut se servir pour terme de comparaison, du membre sain confronté avec le corps malade, et du membre malade confronté avec le membre sain, en ayant soin de mettre en regard, non pas les articualtions du patient, pour juger si le membre sain est dissemblable (ảvó $\mu \alpha \lambda o v)$ du membre blessé...» On voit que la «justice» du corps, ici, est l'égalité, la ressemblance entre les membres latéraux, l'équilibre. Ce passage n'a pas manqué de retenir Erotien ${ }^{33}$ et Galien ${ }^{34}$. En vérité, cet équilibre du corps ne renvoie pas à un modèle transcendant, mais regarde le corps de chaque individu que doit considérer le praticien.

Galien a une toute autre idée de la «justice» de la nature, quand il écrit, à propos d'Hippocrate encore: «Si jamais un créateur a fait preuve d'une

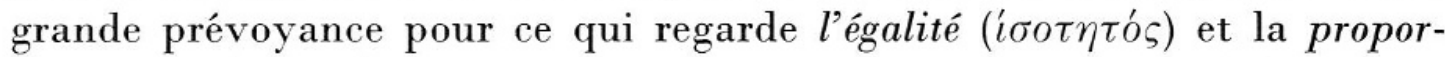
tionnalité ( $\alpha$ v $\left.\alpha \lambda \gamma_{0} \hat{j}_{\alpha \varsigma}\right)$, la nature a aussi donné cette preuve dans la formation du corps des vivants, d'où Hippocrate lui décerne avec raison le nom de juste» ${ }^{35}$.

Le rapport entre la médecine et l'art semble donc d'autant plus naturel et fondé que c'est Galien qui est responsable de la transmission des témoignages les plus complets sur le Canon de Polyclète ${ }^{36}$, et qu'il utilise assez souvent le nom de Polyclète et de Phidias en réfléchissant sur leur technique. D'autre part, en ce qui concerne les «progrès» de la sculpture et de son épanouissement au milieu du $\mathrm{V}^{\mathrm{e}}$ siècle av. J.C. (le Doryphore serait du début de la seconde moitié de ce $\mathrm{V}^{\mathrm{e}}$ siècle), les critiques d'art laissent un peu aller leur plume, à parler du progrès de l'anatomie des sculpteurs, sans trop se demander si ces termes ont un sens. Certains n'hésitent pas à comparer l'artiste de la Renaissance et celui du $\mathrm{V}^{\mathrm{e}}$ siècle, ce qui est une totale absurdité ${ }^{37}$. Mais du moins ces auteurs ont-ils le mérite d'une certaine franchise tandis qu'une espèce de sous-entendu, de «cela-va-de-soi», couvre pudiquement, si l'on peut 
dire, l'«anatomie» des statues grecques. Il est très intéressant, à notre avis, de considérer un peu ce qu'était le corps pour le médecin, que nous dirons grossièrement contemporain, de la Collection hippocratique, même si, et c'est encore plus significatif, beaucoup de ces textes hippocratiques sont très postérieurs à cette période.

La première remarque que l'on doit faire, c'est qu'il n'y a pas de quoi, dans ces écrits, inciter à la méditation artistique, ne disons pas seulement sur la beauté, mais même sur la forme. L'anatomie, au sens de connaissance de l'intérieur du corps, n'est pas une préoccupation majeure du médecin hippocratique, même si, pour employer une expression galénique, il existe, chez Hippocrate, un «mélange de contemplation anatomique». Il ne faut pas en vouloir aux Anciens, dit Galien, de n'avoir pas beaucoup écrit sur l'anatomie ${ }^{38}$. Bien entendu il faudrait nuancer longuement. Mais l'on peut dire sans trop se tromper que le corps hippocratique est un corps fait de plein et de vide, de dur et de mou, de tendu et de lâche, et surtout traversé, irrigué de liquides, humeurs, sueurs, urine, et qui ne possède pas en lui, si j'ose dire, la raison juridique de sa propre structure, comme s'il n'y avait en lui aucune nécessité de sa forme ${ }^{39}$. Evidemment l'on me citera aussitôt ce passage de Nature des os: «Les os donnent au corps le maintien (stasin), la rectitude (orthotêta) et la forme (eidos); les tendons (nerfs), la flexion, la contraction et l'extension; les chairs et la peau la liaison et l'arrangement de tout; les veines, répandues dans tout le corps, le souffle, le flux et le mouvement, les veines qui proviennent nombreuses d'une seule, et cette veine unique, où elle commence et où elle finit je ne sais, car un cercle étant accompli le commencement n'en est pas trouvé.» ${ }^{40} \mathrm{Si}$ intéressant que soit ce passage, il est solitaire et le médecin ne se préoccupe pas généralement des rapports entre la forme externe et l'intérieur du corps.

La beauté n'est pas une préoccupation hippocratique. Le concept du $x^{\prime} \alpha \lambda \lambda \varsigma$, la beauté, n'intervient jamais ${ }^{41}$. Ce corps des médecins est alors surtout fait de profondeurs soupçonnées. La médecine hippocratique, et j'entends bien qu'on trouvera des exceptions, ne s'intéresse pas beaucoup à la forme ni même aux formes. Elle est surtout orientée vers le qualitatif, et ce

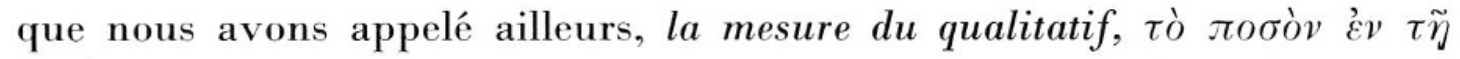

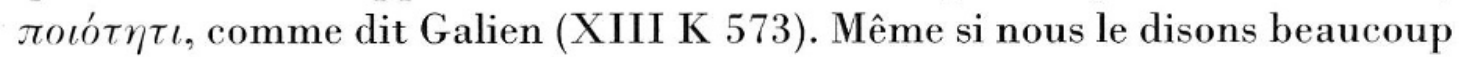
trop vite ici, notre thèse est que le médecin, ni a fortiori l'artiste, ne peut avoir l'idée que la forme du corps naisse de l'intérieur du corps; ni l'un ni l'autre ne peuvent concevoir qu'il y ait une raison nécessaire de cette ligne extérieur qui viendrait des profondeurs du corps. 
L'on peut dire cela autrement. D'une part, il ne semble pas, il n'est pas de preuve, que l'art ait eu à apprendre de la médecine. Il faudra attendre l'époque de Vinci et de Vésale pour que le regard de l'artiste rencontre le regard du médecin et coïncide avec lui. Cela suppose une longue histoire qu'il est très prétentieux de même évoquer dans un si bref article, mais je pense qu'à l'époque de Phidias, ou de Polyclète, la peau que reproduit le statuaire n'a, en soi, légitimement, rien à voir avec le derme du médecin. Je pense que la forme du sculpteur ne suppose aucune nécessité interne, que la ligne n'a aucune raison venue des profondeurs. Sa raison et son droit est tout extérieure et mesurable. Pour le médecin de cette époque le derme n'est autre chose qu'une sorte de sac; la ligne de surface n'est pas ce qui l'intéresse ${ }^{42}$.

L'élément qui donne son «modelé» au corps est, chez le médecin, celui

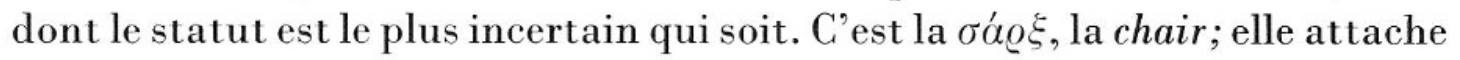
à l'os, elle peut être plus ou moins sèche, elle est recouverte par la peau; elle attire les humeurs, ou les repousse; elle est poreuse; l'air et les liquides la traversent. Mais elle n'a pas de nécessité formelle.

Plus tard, le pseudo-Galien (XIX K 367), lui donnera la définition suivante: «La chair est faite de sang coagulé; elle est humide et chaude et fournit au corps une molle protection.» Rufus d'Ephèse écrit: «La chair est la partie solidifiée qui, dans les viscères, se trouve entre les vaisseaux; c'est en même temps un certain tissu, et un moyen de remplissage entre les mailles des vaisseaux afin qu'il n'y ait point de vide entre eux, puis il y a la chair des muscles, fibreuse et résistante; enfin celle qui se forme sur les plaies ou qu'on trouve dans les cavités des os est une coagulation.» ${ }^{42}$ bis Là encore, il faudrait nuancer; montrer que la chair coagulée est à l'origine de l'articulation du corps. Mais ce qui est surtout important est de percevoir ce rôle de

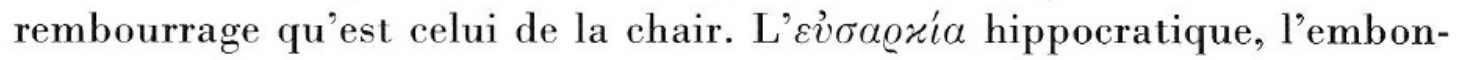
point ${ }^{43}$, si l'on veut, n'a certainement pas une valeur normative. C'est Galien qui commentant, par exemple, l'Aphorisme II, 44 d'Hippocrate ${ }^{44}$ «les personnes qui sont par nature épaisses sont plus exposées à une mort subite que les autres», ${ }^{45}$ dit que le mieux, en effet, est de naître $\varepsilon \ddot{v} \sigma \alpha \varrho \varkappa o v$ (bien en chair, en donnant a bien un sens normatif), c'est-à-dire, écrit-il, $\sigma v \mu \mu \varepsilon \tau \varrho o v$, bien proportionné. Fuchs, qui commente aussi cet aphorisme félicite Galien de donner comme équivalent a $\varepsilon \ddot{v} \sigma \alpha \varrho \varkappa o v, \sigma \dot{v} \mu \mu \varepsilon \tau \varrho o v$, et cite, bien entendu, le quadratum de Celse ${ }^{46}$.

Nous n'avons pas le loisir, en un si court article, d'étudier toutes les nuances que nous devrions apporter à notre proposition. Disons que la statuaire n'avait rien à tirer de la médecine contemporaine, au $\mathrm{V}^{\mathrm{e}}$ siècle av. 
J. C., et bien après sans doute, et que la réflexion esthétique n'est pas un souci de la Collection hippocratique. Or, nous avons vu, avec Galien, combien la pensée de l'art, la réflexion sur le beau et l'acte du sculpteur pénètre la médecine. Il est évident, pour nous, que cette mise en rapport de l'art et de la physiologie n'est possible que par la médiation philosophique. L'idée d'une régulation esthétique de la nature n'a, en effet, rien d'évident ni de spontané. Cette régulation est essentiellement aristotélicienne et stoïcienne.

Aristote d'abord. Il s'agit de régler la question posée par les bœufs à tête d'homme d'Empédocle, celle de la dangereuse spontanéité de la nature. C'est l'idée de l'art qui va servir de paradigme à la conception de la nature. Jamais création naturelle et esthétique ${ }^{47}$ n'ont été rapprochées aussi rigoureusement que dans le livre II de la Physique ${ }^{48}$. Nature et technique sont toutes deux réglées par la cause en vue de quoi, par le projet. La nature est un artiste complet puisque c'est l'artiste qui a en soi son propre principe d'action ${ }^{49}$. Nous ne pouvons ici être que grossièrement allusif. Nous dirons seulement que l'on a expliqué ce livre II de la Physique en accentuant les différences entre la nature et l'art, en marquant les limites de l'art, en renforçant la supériorité de la nature, sans voir que les limites de l'art sont la garantie de la nature. Je dois faire confiance à la nature parce que je vois ce qui se passe dans l'art. Le monstre n'est qu'un raté qui ne met pas en cause le projet. C'est dans la mesure où l'art ne délibère pas, c'est-à-dire qu'il pose son projet et agit en vue de le réaliser, que la nature n'enfante pas n'importe quoi. La nature ne saurait être plus anarchique que l'art. Nous avons l'air de dire que la nature imite l'art, en renversant la proposition «l'art imite la nature». Tel est bien le paradoxe et l'originalité d'Aristote. L'art imite la nature dans la mesure où la nature ressemble à l'art ${ }^{50}$.

Mais c'est peut-être l'idée stö̈cienne de la nature conçue par Zénon comme feu artiste qui est la plus précieuse. «Nous», dit le Stoïcien, par opposition aux Epicuriens, «quand nous disons que la nature forme le monde et le gouverne, nous n'entendons pas que ce soit comme une motte de terre, comme un morceau de pierre,... dont les parties n'ont point de liaison nécessaire les unes avec les autres; nous l'entendons comme dans un arbre, comme dans un animal, où rien ne paraît disposé aveuglément, mais dont les parties sont dans un ordre qui tient de l'art (in quibus nulla temeritas sed ordo apparet, et artis quaedam similitudo $)^{51}$. «La vraie cause, la raison séminale des choses, produit et conserve, et pour produire et conserver, elle procède avec mesure et avec ordre. Elle procède, par conséquent, selon les règles d'un art: art primordial, sur lequel tous les autres arts sont venus ensuite prendre 
modèle, art infaillible, emprunt d'une suprême raison...», écrit très justement Ravaisson ${ }^{52}$. L'idée stoïcienne est précieuse dans la mesure où elle permet d'unir ce que nous appelions la théorie de la re-production et la théorie de la production, grâce à la notion de mélange, de $\varkappa \varrho \tilde{\alpha} \sigma \iota \varsigma \delta$ 'ő $\lambda \omega v$, le logos, la raison compénétrant la matière sans se confondre avec elle et la modelant ${ }^{53}$. Le Stoïcisme, comme on l'a déjà remarqué, et mal gré qu'il en ait, a beaucoup influencé la pensée de Galien.

\section{Conclusion}

Pour en revenir au quadratum de Celse, rien ne peut montrer que le médecin ait pensé un seul instant à Polyclète ni à quelque statue que ce soit; qu'il ait même eu une seule réminiscence esthétique. Mais rien ne peut prouver non plus que les contemporains et successeurs de Celse aient eu tort d'y songer. A l'époque de Celse, l'ambiguïté est possible et plausible. Mais cela dépasse de beaucoup le problème particulier de la phrase de Celse. Il s'agit des rapports entre la santé et la beauté, entre le corps vivant et le corps représenté, et nous avons essayé de montrer, bien trop rapidement, que ces rapports sont beaucoup plus complexes qu'on ne se l'imagine souvent, et que, surtout, il existe une histoire de ces rapports. Entre Polyclète et Hippocrate, pour simplifier outrageusement, il ne pouvait guère exister d'influence possible. Le corps dont s'occupe le statuaire, constitué de parties dont il décide entre elles un rapport harmonieux n'est pas celui que médite le médecin, et qui est plutôt fait de flux que de formes. On pourrait dire, et ce ne serait pas une simple formule, que c'est le sculpteur et non le médecin qui a articulé le corps antique. Il n'est pas, chez Hippocrate, de forme canonique, à laquelle on pourrait rapporter, par comparaison, le corps de tel ou tel patient. Le corps de l'athlète, par exemple, ne saurait être un modèle, la constitution athlétique étant fondamentalement menacée ${ }^{54}$.

C'est Galien qui a mis en rapport de manière significative Polyclète et Hippocrate en réfléchissant sur la création, en essayant de mettre en rapport les deux formes de la création: celle qu'a connue et définie toute l'Antiquité artistique et rhétorique, la création par reproduction, qui suppose la mise en rapport de parties pré-définies ${ }^{55}$; et la création par production, qui suppose l'altération, l'augmentation, et l'assimilation. En fait, on peut espérer, à un degré supérieur, celui de Dieu ou de la Nature, que les deux modèles de la création coïncident et même s'identifient dans une méditation inspirée soit 
d'Aristote soit surtout des Stoïciens. La référence à Polyclète sert à définir la beauté comme relation harmonieuse entre les parties, distinguant l'artiste et le médecin dont la finalité diffère. Elle permet aussi à Galien de se demander s'il y aurait un canon de la santé, c'est-à-dire une symmetria des éléments qui ne saurait être conçue que comme la moyenne d'un mélange, crase moyenne qui devient alors norme ${ }^{56}$. La nature peut aussi être considérée comme un Polyclète accompli, qui saurait harmoniser l'intérieur comme l'extérieur. C'est que, depuis Hippocrate, l'espace intérieur du corps s'est organisé à la vue humaine. Bien entendu, c'est encore un bourbier, comme l'écrit Galien, en comparaison de l'ordre céleste ${ }^{57}$.

Mais à qui prête attention, une vraie beauté se présente. La nature est un Polyclète de l'intérieur. «Est-il juste d'admirer Polyclète pour la proportion (symmetria) des formes de la statue que l'on appelle canon, et non seulement de ne pas célébrer la nature, mais de lui refuser même toute espèce d'art, quand, loin de se contenter de créer les parties proportionnelles à l'extérieur, comme le font les statuaires, elle a encore établi la même proportion $\grave{a}$ l'intérieur? Ou plutôt Polyclète lui-même n'est-il pas l'imitateur de la nature, des choses qu'il pouvait imiter? Il a imité seulement les choses extérieures dont il a pu voir l'artifice, en commençant par celles qui sont à la portée. Telle est la main.... ${ }^{58}$

En vérité, Galien n'a cessé de réfléchir à une œuvre plus importante que toute la médecine, écrit-il, et qui serait une théologie parfaite ${ }^{59}$. Il faut comprendre que cette théologie, qui établit nécessairement l'utilité, la finalité, la fonction des parties du corps est, pour Galien, ce qu'on pourrait appeler une esthétique fondamentale. L'on ne saurait réduire la réflexion de Galien sur l'art et la beauté à une simple curiosité culturelle. Elle fait le fonds de la pensée galénique. Dieu, artiste suprême, oblige à une réflexion sur l'art à laquelle le médecin ne saurait se soustraire.

\section{Notes}

(Abréviations: $\mathrm{K}=$ (Euvres de Galien, éd. Kühn, Leipzig, 1821-1833, reprint 1965. L $=$ CEuvres complètes d'Hippocrate, éd. Littré, Paris, 1839-1861. D = Euvres de Galien traduites par Ch. Daremberg, Paris, Baillière, 1854. D. K. = Fragmente der Vorsokratiker, éd. Diels-Kranz, 12e éd., 1966. S. V.F. = Stoicorum ueterum fragmenta, éd. von Arnim, Leipzig, Teubner, 1903, reprint 1964.)

${ }^{1} \mathrm{II}, 1,5=$ Marx, p. 46

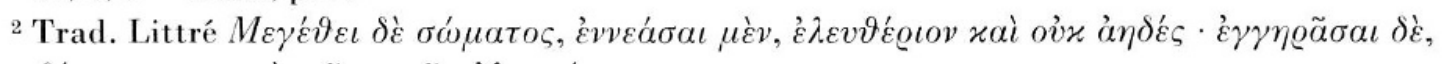

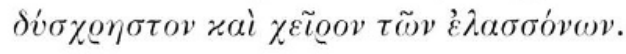


3 Vesp. 20.

${ }^{4}$ De architectura $\mathrm{III}, 1$.

${ }^{4}$ bis XXXIV, 56; trad. H. Le Bonniec, Paris, Belles Lettres, 1953.

5 J.Overbeck, Die antiken Schriftquellen zur Geschichte der bildenden Künste bei den Griechen, Leipzig, 1868 (cité Overbeck), 967.

Un des premiers éditeurs de Celse, Constantin, note dans la marge de son édition en face de

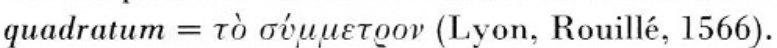

${ }^{6}$ La date de Prorrhétique II est incertaine. Ce livre dans le Corpus hippocratique est très riche et très intéressant, à bien des points de vue. Je serais assez tenté de lui donner une date assez haute. Celse fait allusion à ce passage de Prorrhétique II (De medicina II, $8=$ Marx 67, 20), qu'il transpose ainsi: maximeque lati pectoris atque setosi est, cuiusque cartilago exigua et carnosa est. On voit que l'aspect carré a disparu chez Celse.

7 Nous écartons sciemment toute réflexion sur la valeur du carré, toutes les questions de l'influence pythagoricienne par exemple; cf. sur l'influence de Pythagore sur Polyclète l'article, à notre avis beaucoup trop systématique de J.E. Raven, Polyclitus and Pythagoreanism, in Classical Quarterly, 1951, vol 45, p. 147-152.

${ }^{8}$ Elles sont le sujet d'un livre que nous préparons sur «l'Art et le Vivant».

9 Les noms de Phidias et de Praxitèle apparaissent aussi.

${ }^{10}$ Il n'est pas question de rentrer ici dans la complexe bibliographie sur Polyclète. Nous le ferons ailleurs. Pour une bibliographie récente, on pourra se reporter aux notes de R. Tobin, The Canon of Polykleitos, in American Journal of Archaeology, vol. 79 No 4, oct. 1975, p.307-321. Cette référence n'implique pas que nous acceptions les conclusions de l'auteur. Pour les fragments concernant Polyclète, cf. D. K. I, p.391-393, Overbeck No929-977, R. Bianchi-Bandinelli, Policleto, Florence, 1938.

${ }^{11}$ D. K. I, p. 391 = Galen, On the doctrines of Hippocrates and Plato, first part, books I-V, éd., trad. et comm. de Ph. de Lacy, Berlin, Akademie-Verlag, 1978, p. 309.

12 Cf. notre livre La maladie de l'âme, Paris, Belles Lettres, 1981, p. 295 ss.

13 Nous n'entrons pas, bien sûr, dans les sens de symmetria; cf., pour un examen rapide, J. J. Pollitt, The ancient view of Greek art, criticism, history, and terminology, New Haven and London, Yale University Press, 1974, p. 14-22.

14 I K 559.

15 I K 581.

${ }^{16}$ L'on voit que les citations qui omettent de larges contextes ne sont pas très significatives.

17 I K 566.

18 Ibidem.

19 Il vaudrait mieux parler d'accroissement réglé que de production; cf. par exemple la critique que fait Galien d'Erasistrate qui, célébrant l'art de la nature, pense que les animaux s'accroissent comme un tamis, une chaîne, une corbeille, par application à leur extrémité d'autres parties semblables. «Mais ceci n'est pas un accroissement, ô mortel habile, c'est une production», s'écrie Galien (Fac.nat. II, 3 = II D $256=$ II K 87).

${ }^{20}$ Galien, De usu partium XVII, 1 (II D 203 = IV K 350). Cette phrase vise la «création» selon Asclépiade et Epicure.

21 Galien, Fac.nat. II, 3 = II D $254=$ II K 82 .

${ }^{22}$ Galien, Fac.nat. II, $3=$ II D $254=$ II K 82 .

${ }^{23}$ De usu partium XI, 14, III K $906=$ I D 688, trad. Daremberg. 
${ }^{24}$ Pour avoir voulu faire coïncider l'idée grecque de la création et l'idée sémitique d'une libre création de Dieu, Huarte encourera les foudres de l'Inquisition. Dieu, pense-t-il, ne peut agir lui-même, qu'en suivant les lois qu'il a imposées à la nature; cf. mon article, Fatalisme des tempéraments et liberté spirituelle chez Huarte de San Juan, in Littérature, Médecine et Société No 1, Université de Nantes, 1979, p. 115-159.

${ }_{25}$ De usu partium XI, 13, III K 897 = I D 683, trad. Daremberg; cf. aussi par exemple, XV, 3, IV K $223=$ II D 137.

${ }^{26}$ Cf. la définition de la beauté que donne Cicéron, sans doute issue de Panétius: Pulchretudo enim corporis apta compositione membrorum mouet oculos, ut delectat hoc ipso, quod inter se omnes partes cum quodam lepore consentiunt. (De off. I, 98).

27 De usu partium XI, 13, loc. cit.

${ }^{28}$ C'est aussi l'interprétation la plus faible parmi celles que l'on peut donner du fragment de Polyclète transmis par Plutarque (D.K. I, 392): «Le plus difficile dans l'œuvre, c'est quand l'argile arrive à l'ongle». Ce n'est évidemment qu'une des traductions possibles; et, je le répète, une des interprétations les plus faibles, bien qu'on trouve encore pire.

29 De usu partium XVI, 1, IV K 264 = II D 158; cf. aussi I, 22.

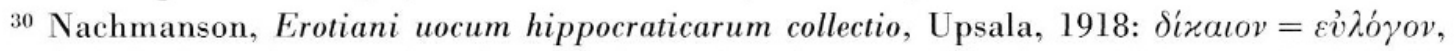

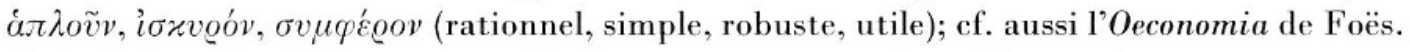

31 Articulations 11; cf. Pétrequin, p. 89-91, et son commentaire p.89, note 4.

32 Articulations 62, Pétrequin, p. 470.

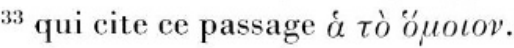

${ }^{34}$ Comm. I, 33, XVIII A K 369, qui donne a díxaıov le sens d'íov.

${ }^{35}$ De usu partium II, 16, III K 158 = I D 209, trad. Daremberg. Ce n'est qu'un exemple. Si nous prenions les sens de symmetros et symmetria dans la Collection hippocratique, nous verrions qu'il est bien difficile de donner à ces termes un sens normatif prégnant.

36 Overbeck No $958-959$.

37 Cf. L'art grec par A.de Ridder et W. Deonna, Paris, La Renaissance du Livre, 1924, p. 237: «Les Grecs étudient l'anatomie en artistes et non en hommes de science; ils éprouvent une émotion esthétique à l'aspect des beaux membres et de leurs jeux harmonieux; on croit les entendre dire, comme plus tard l'artiste de la Renaissance: ‘tu auras du plaisir à dessiner les vertèbres, car elles sont magnifiques; tu dessineras alors l'os qui est placé entre les deux branches, il est très beaù...».

${ }^{38}$ II K 280. Daremberg, comparant l'anatomie homérique et l'anatomie hippocratique, écrit: «Dans Hippocrate, la nomenclature des os est presque aussi indécise que dans Homère, et plus d'une partie du corps n'y est pas mieux décrite.» cf. Histoire des sciences médicales, Paris, Baillière, 1870, t. I, p. 78, note 1.

${ }^{39}$ Par ex. l'intérieur du corps humain, c'est du vide et du plein (De arte 10, VI L 17). Il est puissances et figures (Ancienne médecine 22, I L 627) etc.

40 IX L 182, trad. Littré. Ce passage de Nature des os est ancien et est connu de Galien sous le titre de «suite au Mochlique».

${ }^{41}$ L'on ne dira jamais assez le bien que l'on doit penser de la Concordance des æuvres hippocratiques éditée par G. Maloney et Winnie Frohn, avec la collaboration de P. Potter, 1984.

42 Certes la comparaison entre le sculpteur et le vivant se rencontre très tôt, puisque nous avons au moins le passage de Régime I, 21. «Les sculpteurs imitent le corps, sauf l'âme, et ne font 
pas d'objets pourvus de raison. Ils utilisent l'eau et la terre, séchant ce qui est humide, et mouillant ce qui est sec. Ils enlèvent ce qui est en trop, ajoutent là où il manque, développant du minimum au maximum. L'homme subit le même traitement; il se développe du minimum au maximum, enlevant ce qui est en trop, ajoutant là où il manque, mouillant ce qui est sec et séchant ce qui humide.» Trad. R.Joly. Dans ses Recherches sur le traité hippocratique ‘Du régimes, Paris-Liège, 1961, p.59, R. Joly rapproche de Xén., Mém. III, 10 , à propos de la question de la traduction par les arts plastiques de la vie de l'âme. Nous étudierons ailleurs systématiquement ce type de comparaison.

42 bis $D u$ nom des parties du corps, in Rufus, éd. Daremberg-Ruelle, Paris, Baillière, 1879, p. 164.

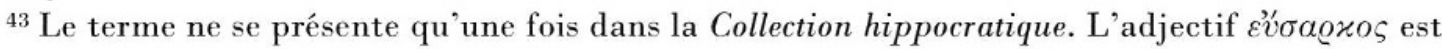
employé 15 fois.

44 XVII B K 547.

45 IV L 482.

${ }^{46}$ Commentaire des Aphorismes d'Hippocrate, Paris, 1545, p. 83.

47 Il faut entendre esthétique au sens général de technique, de production de l'artefact. Aristote cite ensemble le médecin, l'architecte, le sculpteur...

48 Physique B 299 b.

${ }^{49}$ Cf. Métaphysique 3.1070 a 7 .

${ }^{50}$ Cf. 194 a 21 et 199 a 16. C'est ce que signifie profondément l'exemple du médecin de soimême en 199 b 30 .

51 S.V.F. I, 120, 171; II, 423, 774. Sur la nature conçue comme un feu artiste, ef. surtout Cicéron, De natura deorum II, 57, 58 et les notes ad loc. de Stanley Pease dans son admirable édition.

52 Mémoire sur le Stoücisme, in Mémoires de l'Académie des Inscriptions et Belles Lettres, tome XXI' ${ }^{\mathrm{e}}, 1857$, p. 89.

${ }^{53}$ Cette idée stoïcienne de la nature industrieuse et habile vient compléter l'idée de justice dans le passage de Galien que nous commentions précédemment. (De usu partium 16, 1): «Mais si l'on voit ces organes se diriger vers chaque partie en toute sérénité, nous proclamerons la nature non pas seulement juste, mais encore ingénieuse et habile.» (trad. Daremberg - II D

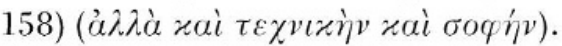

${ }_{54}$ Cf. Aphorismes I, 3, IV L 458. De alimento 34, éd. R. Joly, Paris, Belles Lettres, 1982, p. 145.

${ }_{55}$ Sur la création artistique lire le livre indispensable d'Alain Michel, La parole et la beauté, Paris, Belle Lettres, 1982.

${ }^{56}$ Cela, nous l'avons dit, a pour contrecoup un gauchissement du sens du Canon de Polyclète, et rend très fragile l'interprétation de certains qui ont transporté sur le Canon polyclitéen une idée de moyenne qui vient de la symmetria des éléments et non des parties.

${ }^{57}$ De usu partium XVII, 1, IV K $359=$ II D 207.

${ }_{58}$ De usu partium XVII, 1, IV K 352 = II D 204. 


\section{Summary}

In his «De medicina» Celsus tells us that the body must be quadratus. But what is the meaning of this word? Does it indicate a formal standard, even an aesthetic pattern, as the Canon of Polyclitus, which was square? The tradition seems to have believed in this interpretation. In fact, this paper tries to point to the fact that medicine and theory of art are not naturally connected. Polyclitus and Hippocrates, to speak roughly, are not interested in the same body. Galen, considering Polyclitus' Canon, conceives God as a superior artist, who can conciliate two aspects of creation: reproduction (mimesis), and production. For Galen, the foundation of medicine is what he calls a true theology, in fact the rules of Nature's aesthetics. But it could be conceived only after Aristotle, and above all, the Stoic theory of Nature as a creative fire.

Jackie Pigeaud

2 , avenue de la Berthelotière

F-44700 Orvault (France) 